\title{
Radiative Heat Transfer with MHD Free Convection Flow over a Stretching Porous Sheet in Presence of Heat Source Subjected to Power Law Heat Flux
}

\author{
H. Kumar \\ Department of Information Technology, IBRI College of Technology, IBRI, Oman. \\ hiteshrsharma@gmail.com
}

(Received May 11, 2012; accepted August 20, 2012)

\begin{abstract}
The study of flow and heat transfer in fluid as it passes over a porous surface has attracted considerable scientific attention, particularly in technologies where boundary-layer control is important. Therefore, this paper reports the effects of radiation and heat source over a stretching surface subjected to a power law heat flux, in the presence of transverse magnetic field on two-dimensional boundary layer steady flow and heat transfer of a viscous incompressible fluid. Heat transfer equation is reduced to a linear differential equation using non-dimensional parameters and the exact solution is obtained in the form of confluent hypergeometric function (Kummer's Function) for prescribed power law wall temperature. The effects of the various parameters entering into the problem on the temperature distribution and wall temperature gradient are discussed.
\end{abstract}

Keywords: Heat transfer, Radiation, Porous medium, Magnetic field, Heat source, Kummer's function, Heat flux, Stretching sheet, Suction.

\section{NOMENCLATURE}

$\begin{array}{ll}\mathrm{B}_{0} & \text { transverse magnetic field } \\ u, v & \text { fluid velocities in the } \mathrm{x} \text { and } \mathrm{y} \text { directions } \\ c & \text { stretching rate } \\ x, y & \text { cartesian coordinates } \\ v_{0} & \text { suction velocity across the stretching sheet } \\ \vartheta & \text { kinematic viscosity } \\ K & \text { permeability of the porous medium } \\ \sigma & \text { electrical conductivity } \\ \rho & \text { density of the fluid } \\ T & \text { temperature } \\ k & \text { thermal conductivity } \\ c_{p} & \text { specific heat at constant pressure } \\ q_{r} & \text { radiative heat flux in } \mathrm{y} \text { - direction } \\ Q & \text { heat sink coefficient } \\ T_{\infty} & \text { far field temperature } \\ T_{w} & \text { wall temperature on the sheet at } \mathrm{y}=0\end{array}$

\section{INTRODUCTION}

Studies of heat transfer and flow over moving smooth surfaces impact many technological processes. Examples include aerodynamic extrusion of plastic sheeting and the purification of molten metal to remove non-metallic inclusions. For example, continuous

\author{
A dimensional temperature coefficient \\ $\sigma^{\prime} \quad$ Stefan-Boltzmann constant \\ $\kappa^{*} \quad$ mean absorption coefficient \\ $f(\eta)$ dimensionless velocity function \\ $\eta \quad$ dimensionless coordinate in y direction \\ $\lambda \quad$ permeability parameter \\ $M \quad$ Magnetic parameter \\ $m$ suction parameter \\ $\theta(\eta)$ dimensionless temperature \\ $N$ radiation parameter \\ Pr Prandtl number \\ $\beta \quad$ heat absorption parameter \\ $\xi \quad$ transform parameter \\ ${ }_{1} F_{1} \quad$ Kummer's confluent hypergeometric \\ function \\ $a, b, \alpha, \omega, \gamma, a_{0}, b_{0}$ constants
}

casting, also called strand casting, is the process whereby molten metal is solidified into a "semifinished" billet, bloom, or slab for subsequent rolling in the finishing mills. In this process, molten metal is poured at a controlled rate into a short vertical metal die or mould that is open at both ends. The melt is cooled rapidly by means of water circulation around the 
mould, and the solidified product is withdrawn in a continuous length from the bottom of the mould at a rate consistent with the pouring rate. It is employed mainly for copper, brass, bronze and aluminum and increasingly for cast iron and steel.

In spite of this, in actual practice, boundary layer flow over a stretching sheet must be addressed. For example, in a melt-spinning process, extradite is stretched into a filament or sheet while it is drawn from the die, and the filament or sheet solidifies as it passes through the controlled cooling system.

Sakiadis (1961) first examined the boundary-layer flow of a viscous fluid in the context of plate motion in its own plane. Erickson et al. (1966) and Gupta and Gupta (1977) extended this problem to the case in which suction or blowing existed at the moving surface. Crane (1970) and Mc Cormack and Crane (1973) studied the boundary-layer flow of a Newtonian fluid caused by the stretching of an elastic flat sheet which moves in its own plane with the velocity varying linearly with the distance from a fixed point by the application of a uniform stress. The uniqueness of the exact analytical solutions followed by the two different approaches (Crane, 1970; Mc Cormack and Crane, 1973) was proved simultaneously by Mc Leod and Rajagopal (1987) and Troy et al. (1987). Both the basic flow and the heat transfer problems for linear stretching of the sheet have since been extended in various ways. For example, Afzal and Varshney (1980), Kuiken (1981) and Banks (1983) considered the power law stretching of the plate $\left(u \alpha x^{m}\right)$. Banks and Zaturska (1986) considered the eigen-value problem for boundary-layer over the stretching plate. The hydromagnetic flow and heat transfer case for a linearly stretching plate has been studied by Chakrabarti and Gupta (1979), Chiam (1995) and Abo-Eldahab and Salem (2004). Series solution of unsteady boundary-layer flow that results from a stretching sheet has been considered by Kechil and Hashim (2007) and Liao (2006). Effects of variable thermal conductivity on MHD flow near a stagnation point on a linearly stretching sheet is studied by Sharma and Singh (2009) and study of chemically reactive solute distribution in a steady MHD boundary layer flow over a stretching surface is presented by Uddin et al. (2011).

All these authors have neglected the importance of porous medium; however the analysis of flow through a porous medium has become the core of several scientific and engineering applications. This type of flow is important to a wide range of technical problems, such as flow through packed beds, sedimentation, environmental pollution, centrifugal separation of particles and blood rheology. Moreover, these authors neglected the effects of radiation and internal heat generation. At the same time, however, the study of flow and heat transfer in fluid past a porous surface has attracted considerable scientific attention based on applications in, for example, chemical engineering, where boundary-layer control, transpiration cooling and gaseous diffusion are important. Equally important is the study of heat generation or absorption in moving fluids for problems involving chemical reactions and those concerned with dissociating fluids. Specifically, the effects of heat generation may alter the temperature distribution, consequently affecting the particle deposition rate in nuclear reactors, electronic chips and semiconductor wafers. In fact, the literature is replete with examples of heat transfer in the laminar flow of viscous fluids. For instance, Vajravelu and Hadjinicolaou (1993) studied heat transfer characteristics in the laminar boundary-layer of a viscous fluid over a stretching sheet with viscous dissipation or frictional heating and internal heat generation. Abel et al. (2001) studied convective heat and mass transfer in a viscoelastic fluid flow through a porous medium over a stretching sheet with variable viscosity. Bhargava et al. (2003) have taken the problem of mixed convection micropolar fluid driven by a porous stretching sheet and found the solution by the finite element method. Rashad (2007) has studied the radiative effects on heat transfer from a stretching surface in a porous medium. Finally, Veena et al. (2007) found the non-similar solution for heat and mass transfer flow in an electrically conducting visco-elastic fluid over a stretching sheet embedded in a porous medium.

Moreover, when the temperature of surrounding fluid is high, radiation plays an important role that cannot be ignored (Modest, 2003; Siegel and Howell, 1992). The effects of radiation in steady flows have been reported many studies, including Cess (1966), Arpaci (1968), Cheng and Ozisik (1972), Hasegawa, Echigo and Fakuda (1972), Bankston, Lioyed and Novony (1977), Hossain and Takhar $(1996,1997)$ and Hossain, Pop and Rees (1998). For an impulsively started infinite vertical isothermal plate, Ganesan, Loganathan and Soundalgekar (2001) studied the effects of radiation and free convection by using Rosseland approximation (Brewster, 1992). Recently, Kumar (2009) has studied radiative heat transfer with hydromagnetic flow and viscous dissipation over a stretching surface in the presence of variable heat flux. This paper reports the effects of radiation and heat source over a stretching surface which is subjected to a power law heat flux, in the presence of transverse magnetic field.

\section{FORMULATION AND SOlUTION OF ProblRM}

The present problem is based on a steady twodimensional incompressible viscous laminar flow caused by a stretching sheet, of an electrically conducting fluid under the action of a transverse magnetic field $\mathrm{B}_{0}$, placed in quiescent fluid (Newtonian) in presence of radiation and heat source. The flow is assumed to be in the $\mathrm{x}$-direction which is chosen along the sheet and the y-axis perpendicular to it. The sheet issues from a thin slit at the origin $(0,0)$. It is assumed that the speed of a point on the plate is proportional to its distance from the slit, Fig. 1, and that the boundary-layer approximations are still applicable. In writing the following equations, it is assumed that the external electric field and the electric field resulting from the polarization of charges are negligible. Under these conditions, equations of momentum and energy are:

$\frac{\partial u}{\partial x}+\frac{\partial v}{\partial y}=0$ 


$$
\begin{aligned}
& u \frac{\partial u}{\partial x}+v \frac{\partial u}{\partial y}=\vartheta \frac{\partial^{2} u}{\partial y^{2}}-\frac{\vartheta}{K} u-\frac{\sigma B_{0}^{2} u}{\rho} \\
& u \frac{\partial T}{\partial x}+v \frac{\partial T}{\partial y}=\frac{k}{\rho c_{p}} \frac{\partial^{2} T}{\partial y^{2}}-\frac{1}{\rho c_{p}} \frac{\partial q_{r}}{\partial y} \\
& +\frac{Q}{\rho c_{p}}\left(T_{\infty}-T\right)
\end{aligned}
$$

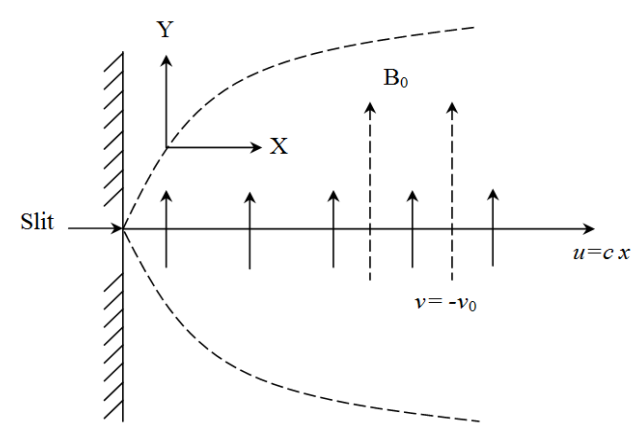

Fig. 1. Boundary-layer flow over a stretching porous surface.

The last term in the RHS of Eq. (3) denotes the heat generation varying directly with the temperature.

The appropriate boundary conditions for the problem are

$$
\begin{array}{lcrl}
\mathrm{u} & =\mathrm{cx}, \quad \mathrm{v}=-v_{0}, \quad \text { at } \quad \mathrm{y}=0 \\
\mathrm{u} & =0, \quad \text { as } & \mathrm{y} \rightarrow \infty
\end{array}
$$

where $\mathrm{c}$ is a positive stretching constant.

In addition, boundary conditions on the temperature are as follows:

$$
\begin{aligned}
& \frac{\partial T}{\partial y}=A x^{2} \text { at } \quad \mathrm{y}=0 \\
& T=T_{\infty} \quad \text { as } \quad \mathrm{y} \rightarrow \infty
\end{aligned}
$$

We assume the Rosselanda approximation (Brewster, 1992) for radiative heat flux, which leads to $q_{r}=-\frac{4 \sigma^{\prime}}{3 \kappa^{*}} \frac{\partial T^{4}}{\partial y}$.

where $\sigma^{\prime}$ is the Stefan-Boltzmann constant and $\kappa^{*}$ is the mean absorption coefficient.

If the temperature differences within the flow are sufficiently small such that $T^{4}$ may be expressed as a linear function of the temperature, then the Taylor series for $T^{4}$ about $T_{\infty}$, after ignoring higher order terms, is given by $T^{4}=4 T_{\infty}^{3} T-3 T_{\infty}^{4}$.

The solution of Eq. (1) and Eq. (2), satisfying the boundary conditions Eq. (4) is as follows:

$$
\begin{aligned}
u & =c x f^{\prime}(\eta), \\
v & =-\sqrt{\vartheta c} f(\eta), \\
\eta & =\sqrt{\frac{c}{\vartheta}} y
\end{aligned}
$$

where prime denotes differentiations with respect to ' $\eta$ ' and

$$
\begin{aligned}
& f(\eta)=a+b e^{-\alpha \eta} \\
& a=\frac{\alpha^{2}-\lambda-\mathrm{M}}{\alpha}, b=-1 / \alpha \\
& \alpha=\frac{m+\sqrt{m^{2}+4(1+\lambda+M)}}{2}
\end{aligned}
$$

where, $\quad \lambda=\vartheta / c K, \quad m=v_{0} / \sqrt{\vartheta c} \quad$ and $\mathrm{M}=\sigma \mathrm{B}_{0}^{2} / \rho c$

Magnetic parameter.

In order to solve Eq. (3), considering boundary conditions Eq. (5), we assume the dimensionless temperature $\theta(\eta)$ as

$T=T_{\infty}+A \sqrt{\frac{\vartheta}{c}} x^{2} \theta(\eta)$

On using Eqs. (6)-(8), Eq. (3) takes the following form:

$$
(1+4 / 3 N) \theta^{\prime \prime}+\operatorname{Pr} f \theta^{\prime}-2 \operatorname{Pr} f^{\prime} \theta-\beta \theta=0
$$

subject to the boundary conditions

$$
\theta^{\prime}=1 \quad \text { at } \quad \eta=0
$$

$\theta(\infty)=0$

where, $N=\frac{\kappa^{*} k}{4 \sigma T_{\infty}^{3}}, \operatorname{Pr}=\frac{\rho \vartheta c_{p}}{k}$ and $\beta=\vartheta Q / k c$.

If we assume $(1+4 / 3 N)=\omega$, then to obtain the solution of Eq. (9), we introduce a new variable $\xi$ as follows:

$$
\xi=-\frac{\operatorname{Pr}}{\alpha^{2} \omega} e^{-\alpha \eta}
$$

Hence Eq. (9) reduces to

$$
\begin{aligned}
& \xi \frac{d^{2} \theta}{d \xi^{2}}+\left(1-\operatorname{Pr}\left(\frac{1}{\omega}-\frac{(\lambda+M)}{\alpha^{2} \omega}\right)-\xi\right) \frac{d \theta}{d \xi} \\
& +\left(2-\frac{\beta}{\alpha^{2} \omega} \xi^{-1}\right) \theta=0
\end{aligned}
$$

The corresponding boundary conditions are

$$
\theta^{\prime}\left(\xi=-\operatorname{Pr} / \alpha^{2}\right)=\alpha / \operatorname{Pr} \text { and } \theta(\xi=0)=0
$$

Solution of the hypergeometric equation, one may refer to Erdélyi (1953),

$$
x \frac{d^{2} y}{d x^{2}}+\left(b^{\prime}+a^{\prime} x\right) \frac{d y}{d x}+\left(\beta^{\prime}+\alpha^{\prime} x+\frac{\gamma^{\prime}}{x}\right) y=0
$$

is $y=x^{-\frac{b^{\prime}}{2}} e^{-\frac{a^{\prime} x}{2}} W(k, \mu, \zeta)$

where, $k=\left(\beta^{\prime}-\frac{a^{\prime} b^{\prime}}{2}\right)\left(a^{\prime 2}-4 \alpha^{\prime}\right)^{-1 / 2}$,

$$
\left.\mu=\frac{1}{2}\left[\left(b^{\prime}-1\right)^{2}-4 \gamma^{\prime}\right]^{-1 / 2}, \zeta=\left(a^{\prime 2}-4 \alpha^{\prime}\right)^{1 / 2} x\right]
$$


Applying the above method the solution of Eq. (12) satisfying boundary conditions Eq. (13) in terms of $\eta$ is given by:

$\theta(\eta)=C e^{-\frac{\alpha\left(a_{0}+b_{0}\right) \eta}{2}}$
$\times{ }_{1} F_{1}\left[\frac{a_{0}+b_{0}-4}{2} ; 1+b_{0} ;-\frac{\operatorname{Pr}}{\alpha^{2} \omega} e^{-\alpha \eta}\right]$

where, $\quad a_{0}=\operatorname{Pr}\left(\frac{1}{\omega}-\frac{(\lambda+M)}{\alpha^{2} \omega}\right), \quad \gamma=\beta / \alpha^{2} \omega$,

$b_{0}=\left[\operatorname{Pr}^{2}\left(\frac{1}{\omega}-\frac{(\lambda+M)}{\alpha^{2} \omega}\right)^{2}+4 \gamma\right]^{1 / 2}$,

$L_{1}=\left(\frac{\operatorname{Pr}}{\alpha \omega}\right)\left[\frac{a_{0}+b_{0}-4}{2\left(1+b_{0}\right)}\right] \times{ }_{1} F_{1}\left[\frac{a_{0}+b_{0}-2}{2} ; 2+b_{0} ;-\frac{\operatorname{Pr}}{\alpha^{2} \omega}\right]$

,$L_{2}=\frac{\alpha\left(a_{0}+b_{0}\right)}{2} \times{ }_{1} F_{1}\left[\frac{a_{0}+b_{0}-4}{2} ; 1+b_{0} ;-\frac{\operatorname{Pr}}{\alpha^{2} \omega}\right]$

and $C=1 /\left(L_{1}-L_{2}\right)$.

The recovery temperature at the stretching plate is given by:

$$
\theta(0)=C_{1} F_{1}\left[\frac{a_{0}+b_{0}-4}{2} ; 1+b_{0} ;-\frac{\operatorname{Pr}}{\alpha^{2} \omega}\right]
$$

\section{RESULTS AND DISCUSSION}

The effects of radiation and heat sink over a stretching surface in the presence of transverse magnetic field, caused by stretching a porous wall are presented graphically. The analytical solutions are obtained in terms Kummer's function (on using the theory of Whittaker function) and the numerical computations of those results obtained by using FORTRAN, are shown in figures.

The dimensionless temperature distribution $\theta(\eta)$ is plotted against $\eta$ for different values of parameters $\mathrm{M}, \lambda, \operatorname{Pr}, \beta, \mathrm{m}$ and $\omega$ in Fig. 2 to Fig. 6, respectively. It has been shown that $\theta(\eta)$ increases with $\operatorname{Pr}$ or $\beta$ or $\mathrm{m}$ increases. Temperature rise due to heat flux impinging on the plate and suction parameter increases with temperature, due to hot suction of fluid. The presence of a heat source in the boundary layer generates energy, which causes the temperature of the fluid to increase. Whereas $\theta(\eta)$ decreases as $\mathrm{M}$ or $\lambda$ or $\omega$ increases. Increasing permeability reduces the presence of solid particles in the medium, thereby reducing the conduction heat transfer which affects the convection transfer process. Temperature decreases as $\omega$ increases (or $\mathrm{N}$ decreases) as it can be seen that an increase in the thermal radiation parameter produces significant increases in the thermal condition of the fluid and its thermal boundary layer. As magnetic field parameter M increases; the Lorentz force, which opposes the flow also increases and leads to an enhanced deceleration of the flow. This result qualitatively agrees with the expectations, since the magnetic field exerts a retarding force on the free convection flow.

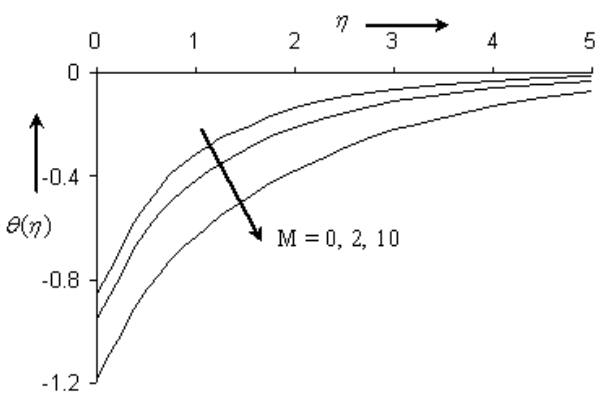

Fig. 2. Dimensionless temperature against $\eta$ for different values of $M$ with $\lambda=1, \mathrm{~m}=0.2, \operatorname{Pr}=1, \beta=0.1$ and $\omega=1.27$

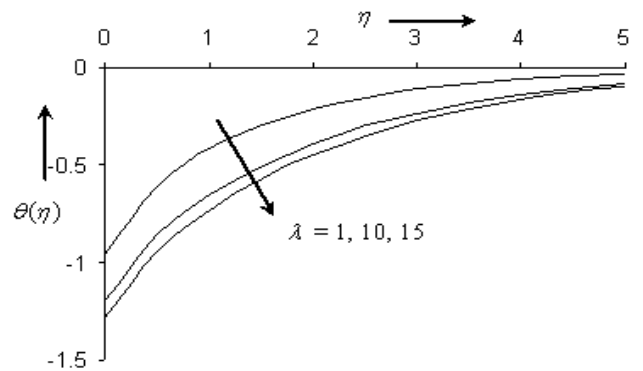

Fig. 3. Dimensionless temperature against $\eta$ for different values of $\lambda$ with $\mathrm{M}=2, \mathrm{~m}=0.2, \operatorname{Pr}=1, \beta=0.1$ and $\omega=1.27$

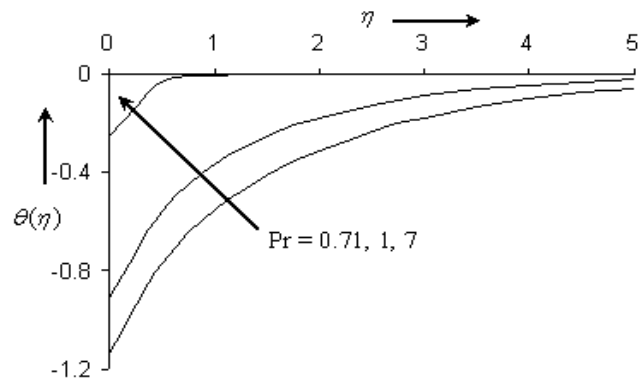

Fig. 4. Dimensionless temperature against $\eta$ for different values of $\operatorname{Pr}$ with $\mathrm{M}=1, \mathrm{~m}=0.2, \lambda=1, \beta=0.1$ and $\omega=1.27$

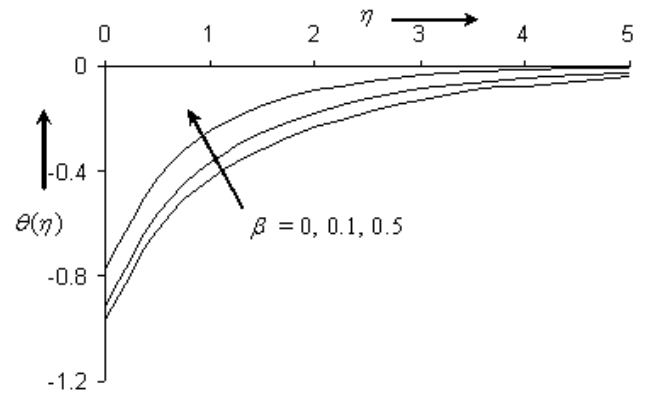

Fig. 5. Dimensionless temperature against $\eta$ for different values of $\beta$ with $\mathrm{M}=1, \mathrm{~m}=0.2, \operatorname{Pr}=1, \lambda=1$ and $\omega=1.27$ 


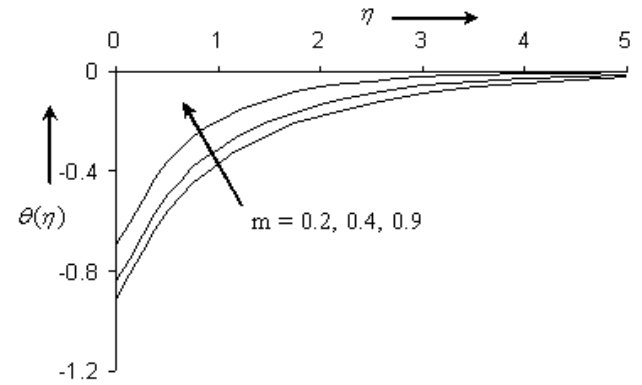

Fig. 6. Dimensionless temperature against $\eta$ for different values of $m$ with $M=1, \beta=0.1, \operatorname{Pr}=1, \lambda=1$

$$
\text { and } \omega=1.27
$$

The recovery temperature $\theta(0)$ is plotted against $\operatorname{Pr}$ for different values of variables, $\mathrm{M}, \lambda, \beta$, $\mathrm{m}$ and $\omega$, as shown in Fig. 7 to Fig. 11, respectively. It is noted that $\theta(0)$ is increased with $\beta$ or $\mathrm{m}$ and it is decreased as $\mathrm{M}$ or $\lambda$ or $\omega$ is increased. The profiles of the function $\theta(0)$ are negative in all the cases. Furthermore, the negative values of the wall temperature gradient for all values of the parameters are indicative of the physical fact that the heat flows from the surface to the ambient fluid.

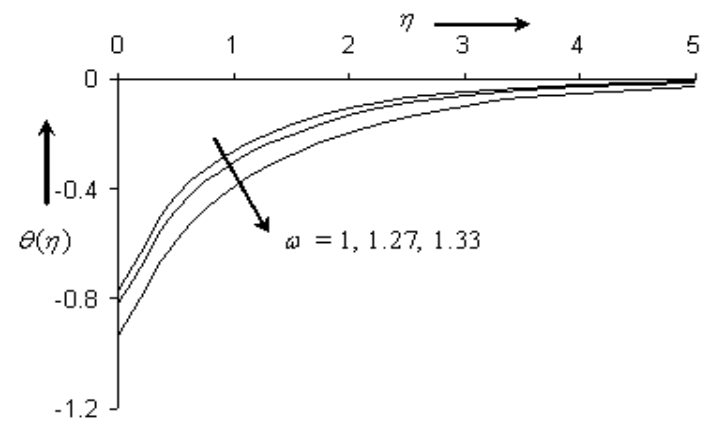

Fig. 7. Dimensionless temperature against $\eta$ for different values of $\omega_{\text {with } M=1, \beta=0.1, \operatorname{Pr}=1, \lambda=1}$ and $\mathrm{m}=0.2$

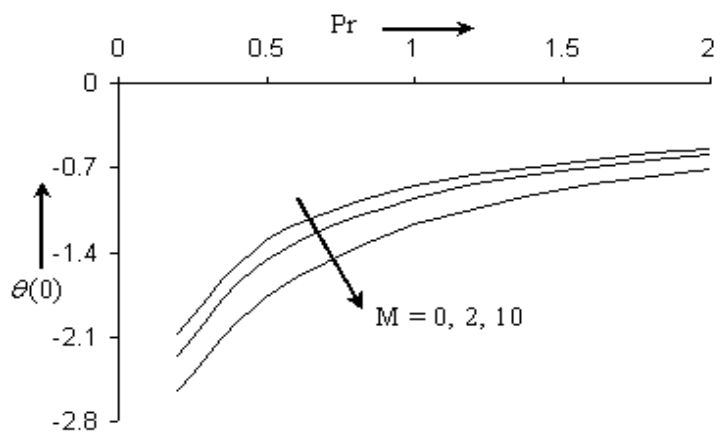

Fig. 8. Dimensionless recovery temperature against $\operatorname{Pr}$ for different values of $\mathrm{M}$ with $\lambda=1, \beta=0.1, \mathrm{~m}=0.2$ and $\omega=1.27$

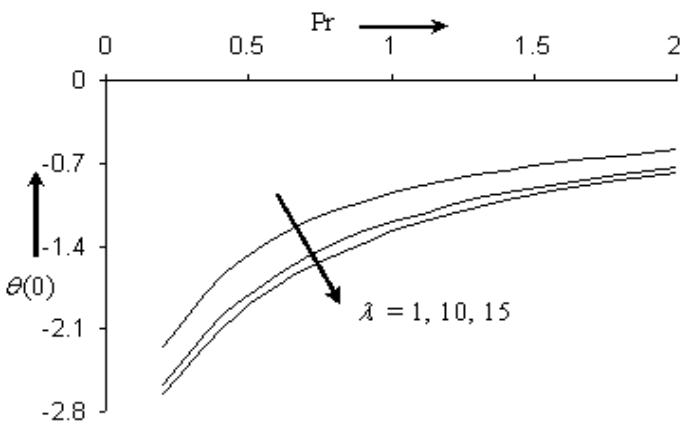

Fig. 9. Dimensionless recovery temperature against $\operatorname{Pr}$ for different values of $\lambda$ with $M=2, \beta=0.1, m=0.2$ and $\omega=1.27$

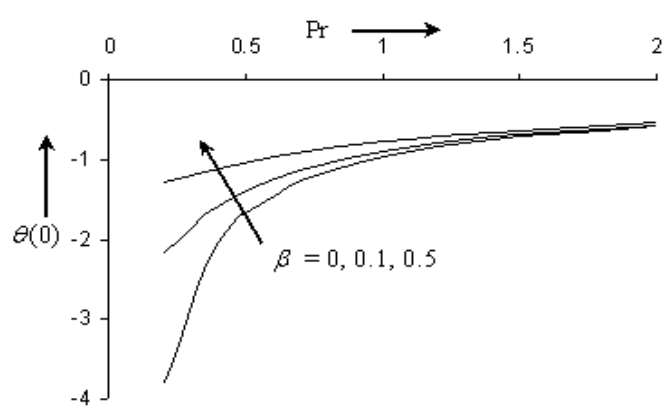

Fig. 10. Dimensionless recovery temperature against $\operatorname{Pr}$ for different values of $\beta$ with $M=1, \lambda=1, \mathrm{~m}=0.2$ and $\omega=1.27$

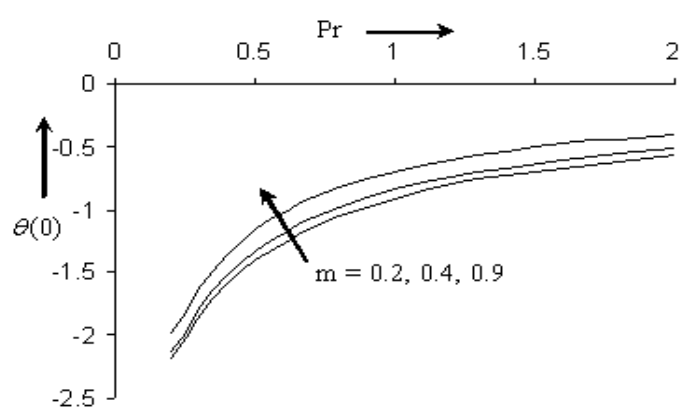

Fig. 11. Dimensionless recovery temperature against $\operatorname{Pr}$ for different values of $\mathrm{m}$ with $\mathrm{M}=1, \lambda=1, \beta=0.1$ and $\omega=1.27$

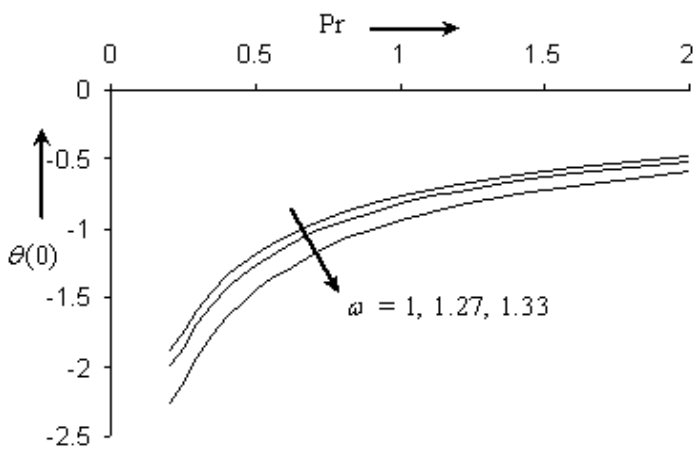

Fig. 12. Dimensionless recovery temperature against $\mathrm{Pr}$ for different values of $\omega$ with $\mathrm{M}=1, \lambda=1, \beta=0.1$ and $\mathrm{m}=0.2$. 


\section{Conclusions}

In the present research, the effects of heat source and porosity on temperature distribution are obtained in Eq. (14) and Eq. (15) which can be interpreted as follows:

1. Radiation and heat source increase the thermal boundary-layer.

2. Porosity and magnetic field decrease the temperature.

3. Radiation and heat source increase the wall temperature gradient.

\section{ACKNOWLEDGEMENTS}

The author is very much thankful to Prof. S. S. Tak, Jai Narain Vyas University, Jodhpur (India) for offering his valuable suggestions and assistance to improve the paper.

\section{REFERENCES}

Abel, M.S., S.K. Khan and K.V. Prasad (2001). Convective heat and mass transfer in a visco-elastic fluid flow through a porous medium over a stretching sheet. Int J Numer Meth Heat Fluid Flow, 11, 779-792.

Abo-Eldahab, E. M. and A. M. Salem (2004). Hall effect on MHD free convection flow of a non-Newtonian power law fluid at a stretching surface. Int Comm Heat Mass Transf, 31, 343-354.

Afzal, N. and I. S. Varshney (1980). The cooling of low heat resistance stretching sheet moving through a fluid. Wirme- und Stoffiibertrag, 14, 289-293.

Arpaci, V. S. (1968). Effects of thermal radiation on the laminar free convection from a heated vertical plate. Int. J. Heat Mass Transfer, 11, 871-881.

Banks, W. H. H. and M. B. Zaturska (1986). Eigen solutions in boundary-layer flow adjacent to a stretching wall. I M A J Appl Math, 36, 263-273.

Banks, W. H. H. (1983). Similarity solutions of the boundary layer equations for stretching wall. $\mathrm{J} \mathrm{Mec}$ Theo Appl, 2, 375-392.

Bankston, J. D., J. R. Lioyed and J. L. Novony (1977). Radiation convection Interaction in an absorbingemitting liquid in natural convection boundary flow. J. Heat Transfer ASME, 99, 125-127.

Bhargava, R., L. Kumar and H. S. Takhar (2003). Finite element solution of mixed convection micropolar flow driven by a porous stretching sheet. Int $J$ Engng Sci, 41, 2161-2178.
Brewster, M. Q. (1992). Thermal Radiative Transfer and Properties. John Wiley and sons Inc.

Cess, R. D. (1966). Interaction of thermal radiation with free convection heat transfer. Int. J. Heat Mass Transfer, 9, 1269-1277.

Chakrabarti, A. and A. S. Gupta (1979). Hydromagnetic flow and heat transfer over a stretching sheet. $Q$ Appl Math, 37, 73-78.

Cheng, E. H. and M. N. Ozisik (1972). Radiation with free convection in an absorbing emitting and scattering medium. Int. J. Heat Mass Transfer, 15, $1243-1252$.

Chiam, T. C. (1995). Hydromagnetic flow over a surface stretching with a power law velocity. Int J Engng Sci, 33, 429-435.

Crane, L. J. (1970). Flow past a stretching plate. ZAMP, $21,645-647$

Erdélyi, A. (1953). Higher Transcendental Functions. Vol.1, Mc Graw-Hill Inc.

Erickson, L. E., L. T. Fan and V. G. Fox (1966). Heat and mass transfer of a moving continuous flat plate with suction or injection. Ind Engng Chem Fundam, $5,19-25$.

Ganesan, P., P. Loganathan and V.M. Soundalgekar (2001). Radiation effects on Flow Past an Impulsively Started Infinite Vertical Plate. Int. J. of Applied Mechanics and Engineering, 6, 719-730.

Gupta, P. S. and A. S. Gupta (1977). Heat and mass transfer on a stretching sheet with suction or blowing. Canad J of Chem Eng, 55, 744-746.

Hasegawa, S., R. Echigo and K. Fakuda (1972). Analytic and experimental studies on simultaneous radiative and free convective heat transfer along a vertical plate. Proc. Jap. Soc. Mech. Eng., 38, 28732883.

Hossain, M. A., I. Pop and D. A. S. Rees (1998). Free convection-radiation interaction from an isothermal plate inclined at a small angle to the horizontal. Acta Mechanica, 127, 63-73.

Hossain, M. A. and H. S. Takhar (1997). Radiationconduction interaction in mixed convection along rotating bodies. Heat and Mass Transfer, 33, 201208. 
Hossain, M.A. and H.S. Takhar (1996). Radiation effects on mixed convection along a vertical plate with uniform surface temperature. Heat and Mass Transfer, 31, 243-248.

Kechil, S. A. and I. Hashim (2007). Series solution for unsteady boundary-layer flows due to impulsively stretching plate. Chinese Physics Letters, 24, 139142.

Kuiken, H. K. (1981). On the boundary layer in fluid mechanics that decay algebraically along stretches on wall that are not vanishingly small. I M A J Appl Math, 27, 387-405.

Kumar, H. (2009). Radiative heat transfer with hydromagnetic flow and viscous dissipation over a stretching surface in the presence of variable heat flux. Thermal Science, 13, 163-169.

Liao, S. J. (2006). Series solutions of unsteady boundary-layer flows over a stretching flat plate. Studies in Applied Mathematics, 117, 239-263.

Mc Cormack, P. D. and L. J. Crane (1973). Physical Fluid Dynamics. Academic Press, New York.

Mc Leod, J. B. and K. R. Rajagopal (1987). On the uniqueness of flow of a Navier- Stokes fluid due to a stretching boundary. Arch Rational Mech Anal, $98,385-393$

Modest, F. (2003). Radiative Heat Transfer. $2^{\text {nd }}$ ed., New York, Academic Press.
Rashad, A. M. (2007). Radiative effect on heat transfer from a stretching surface in a porous medium. Int $J$ Appl Math Mech, 3, 4-23.

Sakiadis, B. C. (1961). Boundary layer behavior on continuous solid surface. AIChE Journal, 7, 26-28.

Sharma, P. R. and G. Singh (2009). Effects of Variable Thermal Conductivity and Heat Source/Sink on MHD Flow Near a Stagnation Point on a Linearly Stretching Sheet. JAFM, 2, 13-21.

Siegel, R. and J. R. Howell (1992). Thermal Radiation Heat Transfer. $3^{\text {rd }}$ ed., Hemisphere, New York.

Troy, W. C., G. B. Ermentrout, J. P. Keener and E. A. Overman-II (1987). Uniqueness of flow of a second order fluid past a stretching surface. $Q$ Appl Math, 44, pp. 753-755.

Uddin, M. S., K. Bhattacharyya, G. C. Layek and W.A. $\mathrm{Pk}$ (2011). Chemically reactive solute distribution in a steady MHD boundary layer flow over a stretching surface. JAFM, 4, 53-58.

Vajravelu, K. and A. Hadjinicolaou (1993). Heat transfer in a viscous fluid over a stretching sheet with viscous dissipation and internal heat generation. Int Comm Heat Mass Transf, 20, 417-430.

Veena, P. H., V. B. Hippargi, V. K. Pravin and S. M. Shahjahan (2007). Non-similar solutions for heat and mass transfer flow in an electrically conducting visco-elastic fluid over a stretching sheet embedded in a porous medium. International Journal of Modern Mathematics, 2, 9-26. 